\title{
Promoting cessation in hospitalized smoking patients: a systematic review
}

\author{
Lígia Menezes do Amaral' \\ Ângela Caroline Dias Albino Destro de Macêdo² \\ (iD) Isabella Oliveira Lanzieri ${ }^{3}$ \\ (D) Rafaela de Oliveira Andrade 3 \\ (iD) Kimber P. Richter ${ }^{4}$ \\ DI Isabel Cristina Gonçalves Leite
}

\begin{abstract}
1. Departamento de Clínica Médica, Faculdade de Medicina, Universidade Federal de Juiz de Fora, Juiz de Fora, MG, Brasil. 2. Bolsista de Iniciação Científica (BIC). Faculdade de Medicina, Universidade Federal de Juiz de Fora, Juiz de Fora, MG, Brasil. 3. Bolsista de Iniciação Científica (Pibic) Faculdade de Medicina, Universidade Federal de Juiz de Fora, Juiz de Fora, MG, Brasil.

4. Department of Population Health. University of Kansas Medical Center, Kansas City, Kansas, USA. 5. Departamento de Saúde Coletiva. Universidade Federal de Juiz de Fora, Juiz de Fora, MG, Brasil.
\end{abstract}

http://dx.doi.org/10.1590/1806-9282.66.6.849

\section{SUMMARY}

OBJECTIVES: The objective of this review was to evaluate high intensity post-discharge follow-up strategies to promote smoking cessation in hospitalized patients.

METHODS: A systematic review was performed, based on the Preferred Reporting Items for Systematic Reviews and Meta-Analyses (PRISMA - P) protocol. The databases used for research were: PubMed, LILACS/BIREME, Scopus, Web of Science, Cochrane and Scielo. The included articles were randomized clinical trials, published from 1990 to 2018, which evaluated in-hospital and post-discharge intervention, and provided a minimum of 30-day care post discharge. The studies aimed to evaluate tobacco cessation.

RESULTS: Fourteen studies were selected for analysis. Across studies, pharmacotherapy was consistently effective for smoking cessation. Communication technologies likewise were consistently effective for cessation and post-discharge access.

CONCLUSION: Effective strategies exist. The challenge for future trials is to determine the best approaches for different clinical contexts, to promote cessation.

KEYWORDS: Tobacco use cessation. Smoking cessation. Patient discharge. Hospitalization. Systematic review.

\section{INTRODUCTION}

In 2018, Datasus ${ }^{1}$ registered more than 11 million hospitalizations in the country, which means that a large contingent of patients spent at least one night in a hospital, including many smokers. This situation configures hospitalization as a valuable opportunity to approach these patients. The post-discharge follow-up of smoking patients is considered a key element for the actions implemented in the hospital environment to be sustained in the home environment. Without the follow-up of smoking patients after hospital discharge, interventions in favor of cessation, initiated during hospitalization, lose effectiveness. However,

DATE OF SUBMISSION: 25-NovApr-2019

DATE OF ACCEPTANCE: 08-Dec-2019

CORRESPONDING AUTHOR: Lígia Menezes do Amaral

Rua Curitiba, 163, casa 4, Jardim da Serra, Juiz de Fora, MG, Brasil - 36038-600

Tel: +55 32 98807-7273

E-mail: ligia.amaral2013@gmail.com 
post-discharge follow-up remains a challenge for hospitals that offer evidence-based smoking treatment ${ }^{2-4}$.

That said, we consider it necessary to evaluate the strategies studied to assist the smoking patient after hospital discharge, seeking to understand which would be the most effective and promising approaches to promote smoking cessation in this group.

There are still few publications with the purpose of evaluating the strategies for approaching smokers after hospital discharge. Brasil occupies a prominent position for its successful tobacco control program, but few national studies address the challenges of post-discharge monitoring.

A meta-analysis that evaluated the approaches to promote the cessation of hospitalized smokers defined high-intensity interventions as those that, in addition to the approach during hospitalization, remained for 30 days after hospital discharge. Interventions classified as high intensity were more effective in promoting cessation $^{3}$. The aim of this study is to contribute to the literature by reviewing studies that evaluated different forms of high intensity approaches in the post-discharge period of smokers to promote cessation.

\section{METHODS}

The studies' eligibility was based on the Preferred Reporting Items for Systematic Reviews and Meta-Analyzes (Prisma-P) protocol. The characteristics evaluated were study design, studied population, types of intervention, presence of a control group and analyzed outcomes ${ }^{5}$.

Randomized clinical trials were selected in order to study interventions in the post-discharge period in smoking patients, with smoking cessation as the main or secondary outcome. Pilot studies were also included, as presented in an important previous review on approaches during the hospitalization period $^{2-4}$.

The studies contemplated interventions in smoking patients initiated during the hospitalization period, or at the time of hospital discharge, with the objective of promoting cessation, extended to post-discharge. Post-discharge follow-up should be maintained for a minimum period of 30 days after the patient leaves the hospital, an intervention considered to be of high intensity by previous meta-analysis ${ }^{6}$.

The studied population was composed of hospitalized smokers, defined here as individuals who smoked in the last 30 days ${ }^{2,3,7}$.
Studies that evaluated the population exclusively of psychiatric patients, who used tobacco in combination with other drugs, and in patients admitted to rehabilitation clinics were excluded.

The control group received the usual care from the various institutions studied.

Studies that presented smoking cessation outcomes, such as self-reported or biochemically proven tobacco abstinence, were included. The period of abstinence assessed after discharge could vary from shortterm, such as seven days after discharge, to long-term, established here as 12 months after discharge. The abstinence to be considered could be punctual, for example, in the last seven days, or continuous, for example, since hospital discharge.

The search strategy adopted was to find articles published in English, Spanish and Portuguese between 1990 and 2018. The choice of the review period was motivated by the development and wide access to new communication resources, such as internet, mobile phones and new communication technologies that started at that time.

The following databases were used for screening: PubMed, Lilacs/Bireme, Scopus, Web of Science, Cochrane and SciELO. To search for gray literature, in an attempt to avoid the non-inclusion of studies due to publication bias, the Open Gray platform was used, in addition to performing a manual search for authors of articles already selected. The following Boolean expressions were used: (TOBACCO USE CESSATION) AND (POST-DISCHARGE) AND (HOSPITALIZATION OR INPATIENT).

For data extraction and review of titles and abstracts, four researchers met in pairs; one of the pairs had the participation of an expert in epidemiology and the other, with a specialist in the treatment of smoking. After the initial search, repeated titles in different databases were excluded. Then, articles that did not meet the proposed acceptability criteria for the review were excluded.

The studies selected in this stage were read in full by the researchers, in order to confirm or discard their eligibility. The decision for inclusion was made by consensus among the four reviewers. The article selection process is described in the flowchart in Figure 1.

The risk of bias was assessed individually in each study according to the Cochrane risk assessment tool (Cochrane Risk of Bias Tool - version 5.1.0), which identifies low, high or uncertain risk of bias, according to the following possibilities: Selection bias, 


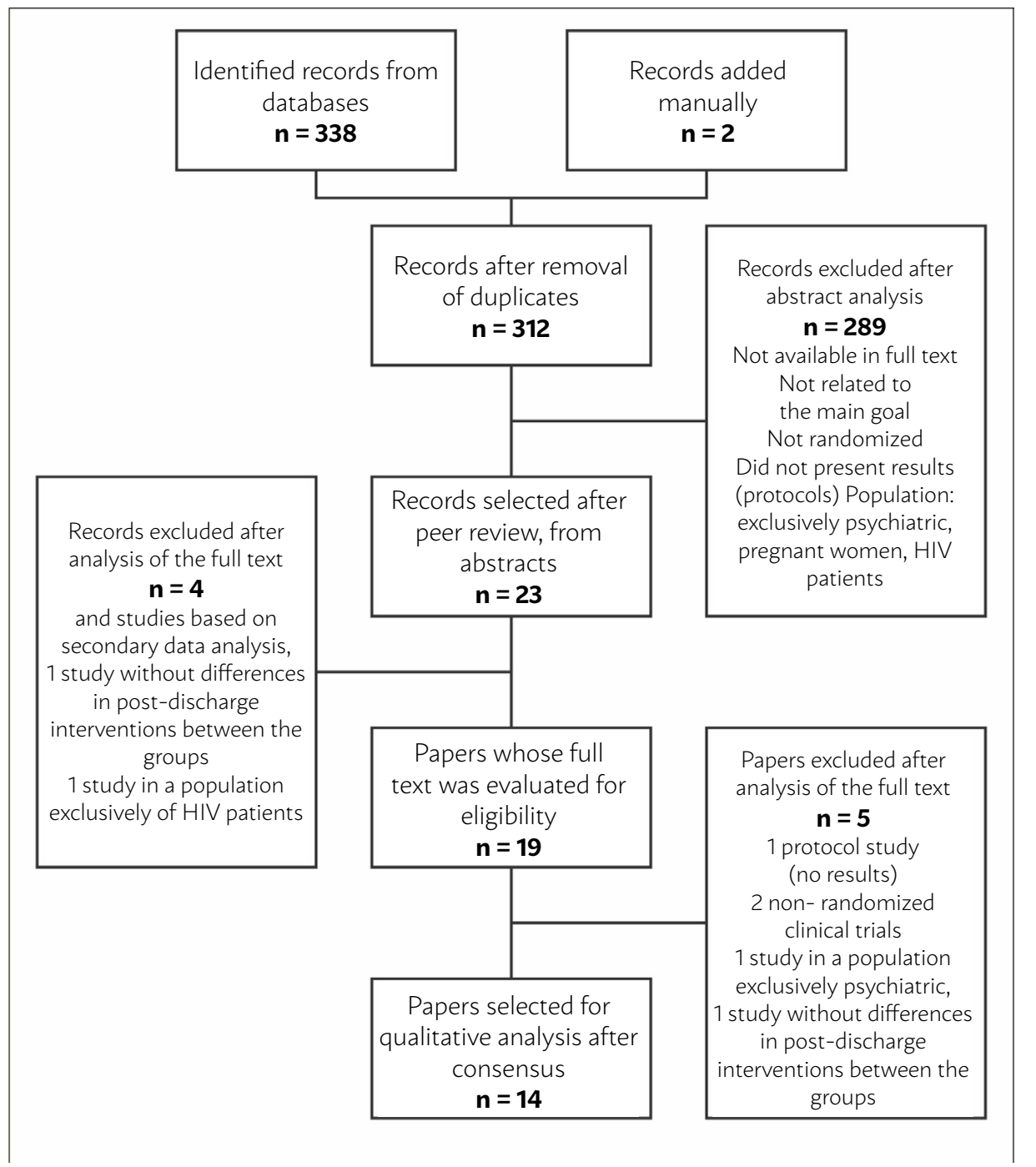

Performance bias (performance), Detection bias, Friction bias, Reporting bias and other biases that do not belong to the aforementioned domains ${ }^{8,9}$.

\section{RESULTS}

The initial selection on the search platforms resulted in 338 articles, two of which were added manually. Among them, 28 were repeated and 293 were excluded after analyzing the title and abstracts and resolving differences between researchers, as they did not meet the established criteria. Five studies were discarded after their complete reading, as they did not fit the search objectives (exclusively psychiatric population, non-randomized studies, uncontrolled studies, future study protocols).

14 studies carried out in the following countries were selected for the review: United States, Canada, Brasil and Australia. The data were extracted from February 1996 to June 2018. Individual data for each study were obtained from publications, as well as their protocols and records on clinical trial registration platforms (U.S. National Institutes of Health Clinical Trials Registry). The characteristics of the selected studies are shown in Table 1.

The follow-up time for the studies ranged from 3 to 12 months. It is worth noting the finding that the current decade has the largest number of publications on the subject, with 11 studies between 2011 and 2018.

The interventions performed during the hospitalization period varied in different publications. The bedside approach, whether for smoking history, demographic data collection or counseling, was a strategy common to all studies. In one of the studies $^{11}$, only the intervention groups received counseling, while the control group received printed informational material.

The pharmacological treatment of smoking, with nicotine replacement during hospitalization, has been used in several studies, with the purpose of reducing abstinence symptoms $s^{6,7,12,13,14,19-21}$.

Also regarding the hospitalization period, several 
studies described the referral to community post-discharge care services (quitlines), and in some studies, the way in which the reference to these services was given, whether assisted or not by the researcher, it was the strategy to be studied ${ }^{7,17}$.

The post-discharge strategies in the intervention group and in the control group are described in Table 1, which also shows the main characteristics of the population of the selected studies, the outcomes related to cessation and the results. The interventions took place at a distance, with contact, in most studies, mediated by communication technologies, with emphasis on telephone calls and interactive voice response (IVR), a technology that allows the interaction between computers and human beings through the using your phone's voice or keypad. One of the studies intervened via text messages. Sometimes e-mail was used to send information after discharge, but it was not the main intervention mechanism. It is also worth mentioning the attempt of several studies to stimulate adherence to quitline programs, intermediating the enrollment of patients in the programs, in order to promote cessation. The follow-up time for the studies ranged from 3 to 12 months.

All the studies analyzed used some type of pharmacotherapy for smoking cessation at some point in the study. Therapy with nicotine replacement (NRT), bupropion and varenicline appear as alternatives for pharmacotherapeutic treatment, with NRT being the most widely used. The data about this are detailed in Table 2. In most studies, there was a balance in the use of pharmacotherapy between the intervention group and the control group. Among the three studies in which the intervention group received pharmacotherapy more frequently than the control group, in one of them pharmacotherapy was part of the proposed intervention ${ }^{6,18,20}$.

The risk of bias was established, in each study, as low $(\mathrm{L})$, high $(\mathrm{H})$ or undetermined $(\mathrm{U})$, considering the following domains: selection, performance, detection, attrition and reporting bias. Table 3 shows the risk of bias in each study. The performance bias was considered high in all studies, in view of the evident difficulty in promoting blindness when offering or receiving interventions, given the nature of the studies. Regarding the detection bias, most studies did not present data regarding the blindness of the outcome evaluators, being, therefore, considered undetermined in most of them. The attrition bias was considered high when the withdrawal of participants was not justified by the authors. In the reporting domain, although some studies have not published a protocol, the outcomes were reported as proposed in the methodology, and therefore, the likelihood of such bias is considered low.

The intention-to-treat analysis was used, considering losses such as still smokers.

\section{DISCUSSION}

A meta-analysis published in $2012^{3}$ categorized interventions to promote the cessation of hospitalized smokers into four groups, according to their intensity. The group considered to be the most intense, and which showed results in the cessation outcomes, was the group with interventions that continued for up to 30 days after discharge. In this review, we analyzed the studies that offered high-intensity approaches to assess the strategies they used.

Behavioral interventions, associated with pharmacological interventions, make up the set of measures to promote the cessation of hospitalized smokers ${ }^{2,22,23}$. The search for those that would be the most effective strategies and the best way to offer them has been the subject of studies, especially in the last decade. The six studies that demonstrated statistically significant differences in termination outcomes had in common the use of some distance communication strategy, such as phone calls, text messages and interactive voice calls, associated with pharmacotherapy ${ }^{10,11,14,18,19,21}$.

The emergence of new communication technologies and the population's growing access to these resources drove the development of strategies and the use of these new tools since the 1990s, supported by the increase in access to telephone sets ${ }^{2,24}$.

Pharmacological treatment with first-line drugs (NRT, bupropion and varenicline) is an important strategy for cessation ${ }^{25}$. Nicotine replacement therapy was the drug strategy highlighted in this review, being common to all studies. Pharmacotherapy had an equivalent prevalence, between intervention and control groups, in most studies, with the exception of three of them ${ }^{15,19,20}$, in which the intervention group received more medication than the control group.

Quitline programs, in which trained counselors provide support for cessation, appear as a consolidated strategy for monitoring smokers after discharge ${ }^{2,22,26}$. The program is part of the standard care adopted by the control group for most studies. The quality of these programs may be one of the explanations for 
TABLE 1. CHARACTERISTICS AND RESULTS OF SELECTED STUDIES

\begin{tabular}{|c|c|c|c|c|c|}
\hline $\begin{array}{l}\text { Study/ } \\
\text { Year of } \\
\text { publication }\end{array}$ & $\begin{array}{l}\text { Country/ } \\
\text { Follow-up } \\
\text { Period }\end{array}$ & $\begin{array}{l}\mathrm{n} \text { and special } \\
\text { characteristics }\end{array}$ & $\begin{array}{l}\text { Group(s)/Interven- } \\
\text { tion/Post-Discharge } \\
\text { Intervention }\end{array}$ & $\begin{array}{l}\text { Control Group/ } \\
\text { Post-Discharge } \\
\text { Intervention }\end{array}$ & $\begin{array}{l}\text { Cessation outcome/Results } \\
\text { (statistically significant difference) }\end{array}$ \\
\hline $\begin{array}{l}\text { Dornellas et } \\
\text { al. } 2000\end{array}$ & $\begin{array}{l}\text { USA } \\
\text { Unicentric } \\
\text { Feb. } 1996 \\
\text { Jan. } 1997\end{array}$ & $\begin{array}{l}100 \\
\text { Patients hos- } \\
\text { pitalized with } \\
\text { acute myocar- } \\
\text { dial infarction }\end{array}$ & $\begin{array}{l}\text { Single group }(n=54) \\
\text { - Bedside counseling } \\
\text { during hospitalization } \\
\text { ( } 20 \text { minutes) and } \\
\text { post-discharge tele- } \\
\text { phone counseling, with } \\
\text { calls at } 1,4,8,12,16,20 \text {, } \\
26 \text { weeks post-discharge }\end{array}$ & $\begin{array}{l}\text { ( } n=46) \\
\text { - Oriented to access } \\
\text { institutional video } \\
\text { during hospital stay } \\
\text { - No interventions } \\
\text { after discharge }\end{array}$ & $\begin{array}{l}\text { Self-reported abstinence for } 7 \text { days and } \\
\text { confirmed by cohabiting, } 6 \text { and } 12 \text { months } \\
\text { after discharge } \\
\text { Results } \\
\text { There was a difference between the } \\
\text { groups } \\
\text { - } 6 \text { months: } \\
\text { - Intervention group }=67 \% \\
\text { - Control group }=43 \%(p<0.05) \\
\text { - } 12 \text { months: } \\
\text { - Intervention group }=55 \% \\
\text { - Control Group }=34 \%(p<0.05)\end{array}$ \\
\hline $\begin{array}{l}\text { Hennrikus } \\
\text { et al. } 2005\end{array}$ & $\begin{array}{l}\text { USA } \\
\text { Multicenter (4 } \\
\text { hospitals) } \\
\text { Jan. } 1997 \\
\text { Jul. } 1999\end{array}$ & $\begin{array}{l}\text { 2,095 } \\
\text { Smokers } \\
\text { hospitalized for } \\
\text { multiple causes }\end{array}$ & $\begin{array}{l}\text { Group } 1(n=703) \\
\text { - Modified standard care } \\
\text { and note highlighted } \\
\text { in the patient's record, } \\
\text { recommending advice } \\
\text { by the assistant team } \\
\text { Group } 2(n=696) \\
\text { - Modified standard } \\
\text { care, note highlighted in } \\
\text { the medical record and } \\
\text { additional telephone } \\
\text { counseling sessions } \\
\text { - } \geq 1-617(90 \%) \\
\text { - } \geq 4-318(63 \%) \\
\text { - } \geq 7-88(13 \%)\end{array}$ & $\begin{array}{l}\text { ( } n=696) \\
\text { - Modified standard } \\
\text { care, } 2 \text { manuals and } \\
\text { referral to com- } \\
\text { munity cessation } \\
\text { programs }\end{array}$ & $\begin{array}{l}\text { - Outcome 1: Self-reported abstinence for } \\
7 \text { days, } 7 \text { days post-discharge } \\
\text { - Outcome 2: Self-reported abstinence for } \\
7 \text { days, } 12 \text { months after discharge } \\
\text { - Outcome 3: Abstinence for } 7 \text { days, } 12 \\
\text { months after discharge confirmed by } \\
\text { salivary cotinine } \\
\text { Results } \\
\text { There was a difference in relation to } \\
\text { outcome } 2 \\
\text { - Outcome } 1: \\
\text { - Group } 1=24 \% \\
\text { - Group } 2=25.2 \% \\
\text { - Control group = } 26 \% \\
\text { - (p>0.05) } \\
\text { - Outcome } 2: \\
\text { - Group } 1=15.2 \% \\
\text { - Group } 2=19.8 \% \\
\text { - Control group = } 15 \% \\
\text { - (p <0.05). } \\
\text { - Outcome } 3: \\
\text { - Group } 1=10 \% \\
\text { - Group } 2=9.9 \% \\
\text { - Control group = } 8.8 \% \\
\text { - (p>0.05) }\end{array}$ \\
\hline $\begin{array}{l}\text { Reid et al. } \\
2007\end{array}$ & $\begin{array}{l}\text { Canada } \\
\text { Pilot } \\
\text { Unicentric } \\
\text { Nov. } 2004 \\
\text { May } 2015\end{array}$ & $\begin{array}{l}100 \\
\text { Smokers } \\
\text { hospitalized } \\
\text { for coronary } \\
\text { disease }\end{array}$ & $\begin{array}{l}\text { Single group }(n=50) \\
\text { Standard care during } \\
\text { hospitalization, RIV } 3, \\
14 \text { and } 30 \text { days post } \\
\text { discharge and additional } \\
\text { counseling as needed }\end{array}$ & $\begin{array}{l}\text { ( } n=50) \\
\text { - Standard care } \\
\text { during hospital- } \\
\text { ization, access to } \\
\text { NRT and printed } \\
\text { material. No other } \\
\text { interventions after } \\
\text { discharge }\end{array}$ & $\begin{array}{l}\text { Self-reported abstinence in the last } 7 \text { days, } \\
52 \text { weeks after discharge } \\
\text { Results } \\
\text { There was no difference between groups } \\
\text { Intervention group }=46 \% \\
\text { Control group }=34.7 \% \\
(p=0.25)\end{array}$ \\
\hline $\begin{array}{l}\text { Regan et al. } \\
2011\end{array}$ & $\begin{array}{l}\text { USA } \\
\text { Unicentric } \\
\text { Dec. } 2007 \\
\text { Jul. } 2008\end{array}$ & $\begin{array}{l}738 \\
\text { Smokers } \\
\text { hospitalized for } \\
\text { multiple causes }\end{array}$ & $\begin{array}{l}\text { Single group }(n=368) \\
\text { - IVR ( } 4 \text { times) in the } 30 \\
\text { days after discharge and } \\
\text { possibility of request- } \\
\text { ing a callback by the } \\
\text { counselor }\end{array}$ & $\begin{array}{l}(n=379) \\
\cdot \text { An IVR } 2 \text { weeks } \\
\text { after discharge }\end{array}$ & $\begin{array}{l}\text { Self-report of cessation } 2 \text { and } 12 \text { weeks } \\
\text { after discharge } \\
\text { Results } \\
\text { There was no difference between the } \\
\text { groups studied } \\
\text { - Withdrawal } 2 \text { weeks after discharge } \\
\text { - Intervention group = 39\% } \\
\text { - Control group }=39 \% \\
\text { - RR } 1.02 \\
\text { - Cl }=0.85-1.22 \\
\text { - Abstinence } 12 \text { weeks after discharge } \\
\text { - Intervention group }=29 \% \\
\text { - Control group }=26 \% \\
\text { - RR } 1.11 \\
\text { - Cl }=0.9-1.41\end{array}$ \\
\hline
\end{tabular}




\begin{tabular}{|c|c|c|c|c|c|}
\hline $\begin{array}{l}\text { Study/ } \\
\text { Year of } \\
\text { publication }\end{array}$ & $\begin{array}{l}\text { Country/ } \\
\text { Follow-up } \\
\text { Period }\end{array}$ & $\begin{array}{l}\mathrm{n} \text { and special } \\
\text { characteristics }\end{array}$ & $\begin{array}{l}\text { Group(s)/Interven- } \\
\text { tion/Post-Discharge } \\
\text { Intervention }\end{array}$ & $\begin{array}{l}\text { Control Group/ } \\
\text { Post-Discharge } \\
\text { Intervention }\end{array}$ & $\begin{array}{l}\text { Cessation outcome/Results } \\
\text { (statistically significant difference) }\end{array}$ \\
\hline $\begin{array}{l}\text { Rigotti et al. } \\
2014\end{array}$ & $\begin{array}{l}\text { USA } \\
\text { Unicentric } \\
\text { Aug. } 2010 \\
\text { Apr. } 2012\end{array}$ & $\begin{array}{l}397 \\
\text { Smokers } \\
\text { hospitalized for } \\
\text { multiple causes } \\
\text { that: } \\
\text { - Received } \\
\text { counseling } \\
\text { during hospital- } \\
\text { ization } \\
\text { - Planned to } \\
\text { quit smoking } \\
\text { - Accepted the } \\
\text { pharmacologi- } \\
\text { cal treatment }\end{array}$ & $\begin{array}{l}\text { Single group }(n=198) \\
\text { - Medication for } 30 \\
\text { days, with replacement } \\
\text { twice for up to } 90 \text { days, } \\
5 \text { IVR }(2,14,30,60 \text { and } \\
90 \text { days after discharge) } \\
\text { and fax to the primary } \\
\text { care physician informing } \\
\text { about the treatment }\end{array}$ & $\begin{array}{l}\text { ( } \mathrm{n}=199) \\
\text { - Recommendation } \\
\text { for free call to quit- } \\
\text { line, individualized } \\
\text { recommendation } \\
\text { for medication and } \\
\text { note in the medical } \\
\text { record alerting the } \\
\text { attending physician } \\
\text { about the prescrip- } \\
\text { tion of medication }\end{array}$ & $\begin{array}{l}\text { Biochemically proven abstinence for } 7 \\
\text { days, } 6 \text { months after discharge (cotinine, } \\
\text { or monoxymetry for those using NRT), } \\
\text { self-reported abstinence for } 7 \text { days and } \\
\text { abstinence continues } 2.3 \text { and } 6 \text { months } \\
\text { after discharge } \\
\text { Results } \\
\text { There was a difference between the } \\
\text { groups in the main outcome and in some } \\
\text { secondary outcomes } \\
\text { - Biochemically proven withdrawal: } \\
\text { - Intervention group }=26 \% \\
\text { - Control group }=15 \% \\
\text { - (p=0.009) } \\
\text { - Self-reported abstinence last } 7 \text { days } 6 \\
\text { months after discharge: } \\
\text { - Intervention group }=40.9 \% \\
\text { - Control group }=28.1 \% \\
\text { - (p = 0.008) } \\
\text { - Continuous self-reported abstinence } 6 \\
\text { months after discharge: } \\
\text { - Intervention group }=27.3 \% \\
\text { - Control group }=16.1 \% \\
\text { - ( } p=0.007 \text { ) } \\
\text { - Other outcomes without statistically } \\
\text { significant difference between groups }\end{array}$ \\
\hline $\begin{array}{l}\text { Cummins et } \\
\text { al. } 2016\end{array}$ & $\begin{array}{l}\text { USA } \\
\text { Multicenter } \\
\text { Jun. } 2011 \\
\text { Nov. } 2013\end{array}$ & $\begin{array}{l}1,270 \\
\text { Smoking } \\
\text { patients hos- } \\
\text { pitalized in } 5 \\
\text { hospitals }\end{array}$ & $\begin{array}{l}\text { Group } 1(n=320) \\
\text { - Nicotine patches } \\
\text { Group } 2(n=317) \\
\text { - Nicotine patches and } \\
\text { telephone advice } \\
\text { Group } 3(n=317) \\
\text { - Telephone advice }\end{array}$ & $\begin{array}{l}\text { ( } n=316) \\
\text { - Standard care: } \\
\text { quitline }\end{array}$ & $\begin{array}{l}\text { Self-reported abstinence for } 7 \text { and } 30 \\
\text { days, } 2 \text { and } 6 \text { months after discharge, and } \\
\text { salivary cotinine-confirmed abstinence in } \\
\text { those who reported abstinence for } 7 \text { days, } \\
6 \text { months after discharge. } \\
\text { Result } \\
\text { There was no difference between the in- } \\
\text { tervention groups and the control group or } \\
\text { between the different intervention groups } \\
\text { in any of the analyzed outcomes } \\
\text { Main outcome: } \\
\text { - Abstinence for } 30 \text { days, } 6 \text { months after } \\
\text { discharge: } \\
\text { - Groups without patches = } 18.3 \% \\
\text { - Groups with patches = } 22.8 \% \\
\text { - (p = 0.051) } \\
\text { - Counseling groups = } 21.1 \% \\
\text { - Groups without counseling = } 20.0 \% \\
\text { - (p = 0.65) }\end{array}$ \\
\hline $\begin{array}{l}\text { Fellows et } \\
\text { al. } 2016\end{array}$ & $\begin{array}{l}\text { USA } \\
\text { Multicenter } \\
\text { Nov. } 2011 \\
\text { Nov. } 2013\end{array}$ & $\begin{array}{l}898 \\
\text { Smoking } \\
\text { patients } \\
\text { hospitalized for } \\
\text { multiple causes }\end{array}$ & $\begin{array}{l}\text { Single group ( } n=597) \\
\text { - Approach during } \\
\text { hospitalization } \\
\text { - Proactive reference } \\
\text { for post discharge } \\
\text { assistance } \\
\text { - Pharmacotherapy } \\
\text { (offered according to a } \\
\text { health plan) and } 4 \text { IVR 4, } \\
14,28 \text { and } 49 \text { days after } \\
\text { discharge }\end{array}$ & $\begin{array}{l}\text { ( } \mathrm{n}=301) \\
\text { - Approach during } \\
\text { hospitalization } \\
\text { - Standard care after } \\
\text { discharge: } \\
\text { - Information on } \\
\text { medications and } \\
\text { how to access } \\
\text { quitline. } \\
\text { - A brief follow-up } \\
\text { call to assess ces- } \\
\text { sation }\end{array}$ & $\begin{array}{l}\text { Self-reported abstinence in the last } 30 \\
\text { days, } 6 \text { months after randomization } \\
\text { Result: } \\
\text { There was no difference in cessation } \\
\text { between the two groups in the primary or } \\
\text { secondary outcomes related to cessation } \\
\text { - Intervention group }=24 \% \\
\text { - Control group }=22 \% \\
\cdot(p=0.159)\end{array}$ \\
\hline $\begin{array}{l}\text { Harrington } \\
\text { et al. } 2016\end{array}$ & $\begin{array}{l}\text { USA } \\
\text { Unicentric } \\
\text { Jul. } 2011 \\
\text { May } 2013\end{array}$ & $\begin{array}{l}1,488 \\
\text { Smokers } \\
\text { hospitalized for } \\
\text { multiple causes }\end{array}$ & $\begin{array}{l}\text { Single group }(n=748) \\
\text { - Approach during } \\
\text { hospitalization and time } \\
\text { of discharge (Standard } \\
\text { Care), visit by a team } \\
\text { that guided access, } \\
\text { registration and use of } \\
\text { website with various } \\
\text { information on smoking }\end{array}$ & $\begin{array}{l}\text { ( } \mathrm{n}=740) \\
\text { - Standard care }\end{array}$ & $\begin{array}{l}\text { Self-reported abstinence for } 30 \text { days, } 6 \\
\text { months after discharge } \\
\text { Results: } \\
\text { There was no difference between groups } \\
\text { - Intervention group }=25.8 \% \\
\text { - control group }=24.1 \% \\
\text { - }(p=0.436)\end{array}$ \\
\hline
\end{tabular}




\begin{tabular}{|c|c|c|c|c|c|}
\hline $\begin{array}{l}\text { Study/ } \\
\text { Year of } \\
\text { publication }\end{array}$ & $\begin{array}{l}\text { Country/ } \\
\text { Follow-up } \\
\text { Period }\end{array}$ & $\begin{array}{l}\mathrm{n} \text { and special } \\
\text { characteristics }\end{array}$ & $\begin{array}{l}\text { Group(s)/Interven- } \\
\text { tion/Post-Discharge } \\
\text { Intervention }\end{array}$ & $\begin{array}{l}\text { Control Group/ } \\
\text { Post-Discharge } \\
\text { Intervention }\end{array}$ & $\begin{array}{l}\text { Cessation outcome/Results } \\
\text { (statistically significant difference) }\end{array}$ \\
\hline $\begin{array}{l}\text { Sherman et } \\
\text { al. } 2016\end{array}$ & $\begin{array}{l}\text { USA } \\
\text { Jul. } 2011 \\
\text { Apr. } 2014\end{array}$ & $\begin{array}{l}\text { 1,619 } \\
\text { Smokers } \\
\text { hospitalized } \\
\text { for multiple } \\
\text { causes, with a } \\
\text { high number } \\
\text { of participants } \\
\text { on the street or } \\
\text { in temporary } \\
\text { housing (25\%) } \\
\text { alcohol users } \\
\text { (40\%), and } \\
\text { with mental ill- } \\
\text { ness (50\%) and } \\
\text { use of other } \\
\text { drugs (60\%) }\end{array}$ & $\begin{array}{l}\text { Single group }(n=805) \\
\text { - Post-discharge coun- } \\
\text { seling calls (proactive), } \\
2 \text { weeks after discharge, } \\
\text { other calls } 1,3,7,14,30 \\
\text { and } 42 \text { days after the } \\
\text { first call }\end{array}$ & $\begin{array}{l}\text { ( } n=814 \text { ) } \\
\text { - Referred to the } \\
\text { quitline }\end{array}$ & $\begin{array}{l}\text { Self-reported abstinence for } 30 \text { days, } 2 \\
\text { and } 6 \text { months after discharge } \\
\text { Results: } \\
\text { There was a difference between the } \\
\text { groups } \\
\text { - Withdrawal } 2 \text { months after discharge: } \\
\text { - Intervention group }=29.0 \% \\
\text { - Control group }=20.7 \% \\
\text { - (RR } 1.40 \mathrm{Cl}=1.13,1.73 \text { ) } \\
\text { - Withdrawal } 6 \text { months after discharge: } \\
\text { - Intervention group }=37.4 \% \\
\text { - Control group }=32.5 \% \\
\text { - (RR } 1.19 \mathrm{Cl}=1.01,1.40)\end{array}$ \\
\hline $\begin{array}{l}\text { Richter et al. } \\
\text { 2016\# }\end{array}$ & $\begin{array}{l}\text { USA } \\
\text { Multicenter } \\
\text { Jul. } 2011 \\
\text { Out. } 2014\end{array}$ & $\begin{array}{l}1,054 \\
\text { Smokers } \\
\text { hospitalized for } \\
\text { multiple causes }\end{array}$ & $\begin{array}{l}\text { Single group ( } n=527 \text { ) } \\
\text { - Assessment of } \\
\text { withdrawal symptoms, } \\
\text { adjustment of the NRT } \\
\text { dosage (standard care). } \\
\text { Explanations about the } \\
\text { project. Quitline regis- } \\
\text { tration mediated by the } \\
\text { researcher }\end{array}$ & $\begin{array}{l}\text { ( } \mathrm{n}=527) \\
\text { - Standard Care, } \\
\text { assistance with } \\
\text { cessation (quit plan } \\
\text { + providing medi- } \\
\text { cation prescription } \\
\text { after discharge) and } \\
\text { forwarding via fax to } \\
\text { quitline }\end{array}$ & $\begin{array}{l}\text { - Abstinence in the last } 7 \text { days, self-report- } \\
\text { ed, } 6 \text { months after discharge } \\
\text { - Biochemically confirmed abstinence (co- } \\
\text { tinine, carbon monoxide, proxy) } 6 \text { months } \\
\text { after discharge } \\
\text { Results } \\
\text { There was no difference between the } \\
\text { groups studied } \\
\text { - Self-reported abstinence for } 7 \text { days, } 6 \\
\text { months after discharge: } \\
\text { - Intervention group }=25.4 \% \\
\text { - Control group }=25.3 \% \\
\text { - (p = } 0.88) \\
\text { - Abstinence confirmed for } 7 \text { days } 6 \\
\text { months after discharge (carbon monoxide } \\
\text { and cotinine): } \\
\text { - Intervention group = } 23.7 \% \\
\text { - Control group = } 21.6 \% \\
\text { - Oradj }=1.02 \\
\text { - 95\% Cl = 0.77, } 1.35 \\
\text { - (p = } 0.88 \text { ) } \\
\text { - RR = } 1.02 \\
\text { - } 95 \% \mathrm{Cl}=0.82 ; 1.24\end{array}$ \\
\hline $\begin{array}{l}\text { Rigotti et al. } \\
2016\end{array}$ & $\begin{array}{l}\text { USA } \\
\text { Multicenter } \\
\text { Dec. } 2012 \\
\text { Jul. } 2014\end{array}$ & $\begin{array}{l}\text { 1,359 } \\
\text { Smokers } \\
\text { hospitalized for } \\
\text { multiple causes } \\
\text { who: } \\
\text { - Received } \\
\text { counseling at } \\
\text { hospitalization } \\
\text { - Planned to } \\
\text { quit smoking } \\
\text { - Accepted the } \\
\text { pharmacologi- } \\
\text { cal treatment }\end{array}$ & $\begin{array}{l}\text { Single group ( } \mathrm{n}=681 \text { ) } \\
\text { - Medication for } 30 \\
\text { days, with replacement } \\
\text { twice for up to } 90 \text { days, } \\
5 \text { IVR }(2,12,28,58 \text { and } \\
88 \text { days after discharge) } \\
\text { and possibility to access } \\
\text { a counselor if necessary }\end{array}$ & $\begin{array}{l}\text { ( } \mathrm{n}=678) \\
\text { - Recommendation } \\
\text { for free call to quit- } \\
\text { line }(1-800 \text {-QUIT- } \\
\text { NOW), in- } \\
\text { dividualized } \\
\text { recommendation } \\
\text { for medication } \\
\text { - Note in the med- } \\
\text { ical record alerting } \\
\text { the attending } \\
\text { physician about } \\
\text { the medication } \\
\text { prescription }\end{array}$ & $\begin{array}{l}\text { Biochemically proven abstinence for } 7 \\
\text { days, } 6 \text { months after discharge (cotinine or } \\
\text { monoxymetry for those using NRT) } \\
\text { Self-report abstinence assessment at } 1,3 \\
\text { and } 6 \text { months } \\
\text { Results } \\
\text { There was a difference between groups in } \\
\text { outcome } 2 \\
\text { - Biochemically proven abstinence: } \\
\text { - Intervention group }=17 \% \\
\text { - Control group }=16 \% \\
\text { - ( } p=0.58) \\
\text { - Self-reported abstinence for } 7 \text { days, } 1 \\
\text { month after discharge: } \\
\text { - Intervention group }=43 \% \\
\text { - Control group }=32 \% \\
\text { - ( } p<0.0001) \\
\text { - Self-reported abstinence for } 7 \text { days, } 3 \\
\text { months after discharge: } \\
\text { - Intervention group }=37 \% \\
\text { - Control group }=30 \% \\
\text { - ( } p=0.008) \\
\text { - Self-reported abstinence } 6 \text { months after } \\
\text { discharge: } \\
\text { - Intervention group }=31 \% \\
\text { - Control group }=27 \% \\
\text { - ( } p=0.09 \text { ) }\end{array}$ \\
\hline
\end{tabular}




\begin{tabular}{|c|c|c|c|c|c|}
\hline $\begin{array}{l}\text { Study/ } \\
\text { Year of } \\
\text { publication }\end{array}$ & $\begin{array}{l}\text { Country/ } \\
\text { Follow-up } \\
\text { Period }\end{array}$ & $\begin{array}{l}\mathrm{n} \text { and special } \\
\text { characteristics }\end{array}$ & $\begin{array}{l}\text { Group(s)/Interven- } \\
\text { tion/Post-Discharge } \\
\text { Intervention }\end{array}$ & $\begin{array}{l}\text { Control Group/ } \\
\text { Post-Discharge } \\
\text { Intervention }\end{array}$ & $\begin{array}{l}\text { Cessation outcome/Results } \\
\text { (statistically significant difference) }\end{array}$ \\
\hline $\begin{array}{l}\text { Thomas et } \\
\text { al. } 2016\end{array}$ & $\begin{array}{l}\text { Australia } \\
\text { Multicenter } \\
\text { Apr. } 2012 \\
\text { Jun. } 2014\end{array}$ & $\begin{array}{l}600 \\
\text { Smokers } \\
\text { hospitalized for } \\
\text { multiple causes }\end{array}$ & $\begin{array}{l}\text { Single group ( } n=300 \text { ) } \\
\text { - Behavioral approach ( } 2 \\
\text { sessions in the hospital } \\
\text { and 3rd session } 4 \text { weeks } \\
\text { after discharge). Moti- } \\
\text { vational interview for } \\
\text { those who did not want } \\
\text { to quit smoking. Phar- } \\
\text { macotherapy during } \\
\text { hospitalization and } 1 \\
\text { week after discharge. } \\
\text { Pharmacotherapy for } \\
\text { another } 28 \text { days accord- } \\
\text { ing to availability (PBS). } \\
\text { Impressive information } \\
\text { material, quitline refer- } \\
\text { ence, case summary and } \\
\text { action plan for assistant } \\
\text { physician and commu- } \\
\text { nity pharmacist and } \\
\text { follow-up by telephone } \\
\text { at } 1,6 \text { and } 12 \text { months } \\
\text { after discharge }\end{array}$ & $\begin{array}{l}\text { ( } \mathrm{n}=300 \text { ) } \\
\text { - Standard care for } \\
\text { each hospital: } \\
\text { - Brief intervention } \\
\text { during hospital- } \\
\text { ization } \\
\text { - Available phar- } \\
\text { macotherapy: NRT, } \\
\text { bupropion and } \\
\text { varenicline main- } \\
\text { tained for a period } \\
\text { of } 28 \text { days after } \\
\text { discharge according } \\
\text { to PBS and follow } \\
\text { up by phone at } 1,6 \\
\text { and } 12 \text { months after } \\
\text { discharge }\end{array}$ & 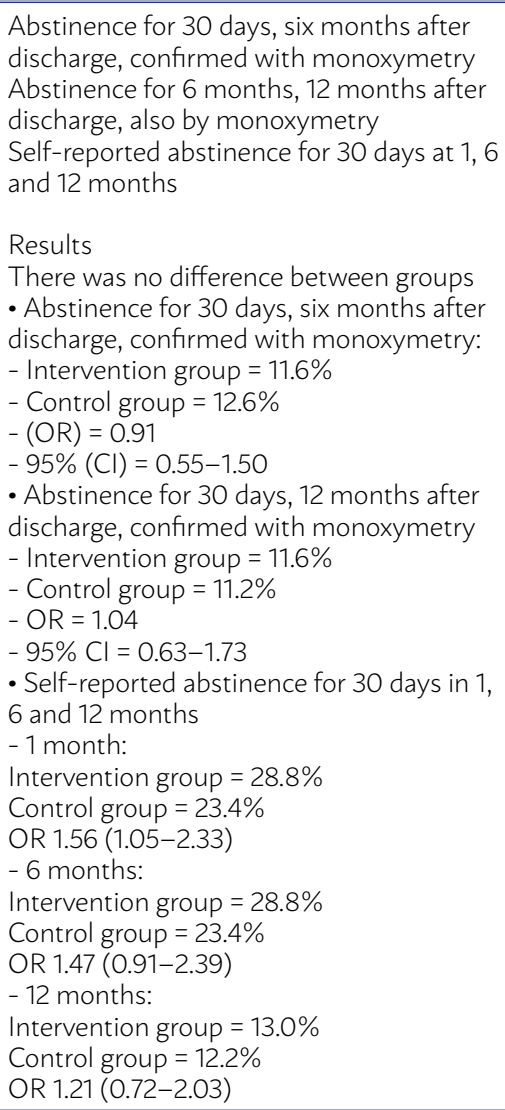 \\
\hline $\begin{array}{l}\text { Busch et al. } \\
2017\end{array}$ & $\begin{array}{l}\text { USA } \\
\text { Pilot } \\
\text { Unicentric } \\
\text { Out. } 2013 \\
\text { Apr. } 2015\end{array}$ & $\begin{array}{l}59 \\
\text { Smoking } \\
\text { patients } \\
\text { hospitalized for } \\
\text { acute coronary } \\
\text { syndrome }\end{array}$ & $\begin{array}{l}\text { Single group }(n=28) \\
\text { - Five counseling ses- } \\
\text { sions in weeks } 1,3,6,9 \\
\text { and } 12 \text { post discharge. } \\
\text { Up to } 4 \text { additional con- } \\
\text { tacts according to need }\end{array}$ & $\begin{array}{l}(n=31) \\
\text { - Received infor- } \\
\text { mative material at } \\
\text { weeks 1, 3, 6, } 9 \text { and } \\
12 \text { after discharge }\end{array}$ & $\begin{array}{l}\text { Abstinence in the last } 7 \text { days, confirmed } \\
\text { by monoxymetry, assessed at the } 12 \text { th and } \\
24 \text { th weeks after discharge. } \\
\text { Result } \\
\text { There was no difference between the } \\
\text { intervention groups and the control group } \\
\text { - } 12 \text { th week: } \\
\text { - Intervention group }=48.0 \% \\
\text { - Control group }=44.8 \% \\
\text { - } 24 \text { th week: } \\
\text { - Intervention group }=45.8 \% \\
\text { - Control group }=42.3 \%\end{array}$ \\
\hline $\begin{array}{l}\text { Cruvinel } \\
2016\end{array}$ & $\begin{array}{l}\text { Brasil } \\
\text { Pilot } \\
\text { Unicentric } \\
\text { Jun. } 2015 \\
\text { Mar. } 2016\end{array}$ & $\begin{array}{l}66 \text { patients } \\
\text { Hospitalized } \\
\text { smokers }\end{array}$ & $\begin{array}{l}\text { Single group }(\mathrm{n}=44) \\
\text { - Brief approach, infor- } \\
\text { mative material printed } \\
\text { during hospitalization, } \\
\text { in addition to NRT for } 4 \\
\text { weeks (standard care) } \\
\text { - And } 1 \text { phone call and } \\
\text { text messages after } \\
\text { discharge }\end{array}$ & $\begin{array}{l}\text { ( } \mathrm{n}=22) \\
\text { - Standard care and } \\
1 \text { follow-up call } 30 \\
\text { days after discharge }\end{array}$ & $\begin{array}{l}\text { Self-reported abstinence in the last } 7 \text { days, } \\
30 \text { days after discharge and } 90 \text { days after } \\
\text { discharge } \\
\text { Abstinence confirmed by monoxymetry } \\
90 \text { days after discharge } \\
\text { Result } \\
\text { There was no difference between the } \\
\text { groups in the } 1 \text { st and } 3 r d \text { outcomes and } \\
\text { there was a difference in the } 2 \text { nd outcome } \\
\text { - } 30 \text { days after discharge: } \\
\text { - Intervention group }=25.0 \% \text { abstinence } \\
\text { - Control group }=9.1 \% \\
\text { - ( } p=0.13) \\
\text { - } 90 \text { days after discharge: } \\
\text { - Intervention group }=31.8 \% \\
\text { - Control group }=9.1 \% \\
\text { - ( } p=0.04) \\
\text { - Abstinence confirmed by monoxymetry: } \\
\text { - Intervention group }=20.5 \% \\
\text { - Control group }=4.5 \% \\
\text { - ( } p=0.09 \text { ) }\end{array}$ \\
\hline
\end{tabular}

NRT: Nicotine Replacement Therapy; IVR: Interactive Voice Response. PBS: Pharmaceutical Benefits Scheme. \#The intervention still occurs at the time of admission, but with the aim of interfering after discharge. 
TABLE 2. USE OF PHARMACOTHERAPY IN THE STUDIES

\begin{tabular}{|c|c|c|c|c|}
\hline Study & Type & Initial Dose & Time & Difference between control and intervention groups \\
\hline Dornelas et al..$^{10}$ & NRT & Not informed & Not informed & $\begin{array}{l}\text { No. Approximately } 24 \% \text { of participants in both } \\
\text { groups. No statistic data }\end{array}$ \\
\hline Hennrikus et al. ${ }^{11}$ & $\begin{array}{l}\text { NRT } \\
\text { Bupropion }\end{array}$ & Not informed & Not informed & No. No additional data (e-mail sent to the author) \\
\hline Reid et al. ${ }^{12}$ & $\begin{array}{l}\text { NRT } \\
\text { Bupropion }\end{array}$ & Not informed & Not informed & $\begin{array}{l}\text { No. NRT: } \\
\text { Intervention Group: } 14 \% \text { Control Group: } 14.3 \% \\
\text { ( } p=0.85 \text { ) } \\
\text { Bupropion: } \\
\text { Intervention Group: } 8 \% \text { Control Group: } 4.1 \% \\
\text { ( } p=0.60 \text { ) }\end{array}$ \\
\hline Regan et al..$^{13}$ & $\begin{array}{l}\text { NRT } \\
\text { Bupropion Varenicline } \\
\text { Other non-specified } \\
\text { mediation }\end{array}$ & Not informed & Not informed & $\begin{array}{l}\text { No } \\
\text { Intervention group: } 69 \% \text { Control group: } 52 \% \\
(p<0.05)\end{array}$ \\
\hline Rigotti et al. ${ }^{14}$ & $\begin{array}{l}\text { NRT, Bupropion } \\
\text { varenicline (Isolated } \\
\text { or combined) }\end{array}$ & Not informed & $\begin{array}{l}\text { Intervention group: } \\
\text { up to } 90 \text { days } \\
\text { Control group: } \\
\text { non-specified }\end{array}$ & $\begin{array}{l}\text { Yes. Intervention group: it was provided. } \\
\text { Intervention group: } 79 \% \\
\text { Control group: } 59 \% \\
\text { RR, } 1.34 \text { [95\% Cl, 1.17-1.54]; }(p<0.001)\end{array}$ \\
\hline Cummins et al. ${ }^{15}$ & NRT & $\begin{array}{l}14 \text { to } 21 \text { mg accord- } \\
\text { ing to number of } \\
\text { cigarettes smoked }\end{array}$ & 08 weeks & $\begin{array}{l}\text { Yes. Medication was part of the strategy in two of the } \\
\text { intervention groups. }\end{array}$ \\
\hline Fellows et al. ${ }^{16}$ & $\begin{array}{l}\text { NRT } \\
\text { Bupropion Varenicline }\end{array}$ & Not informed & Not informed & $\begin{array}{l}\text { No. Intervention group: } 47.0 \% \text { - Control group: } 38.0 \% \\
(p=0.013)\end{array}$ \\
\hline Harrington et..$^{17}$ & $\begin{array}{l}\text { NRT } \\
\text { Bupropion }\end{array}$ & Not informed & Not informed & $\begin{array}{l}\text { No. Intervention group: } 25.9 \% \text { Control group: } 26.0 \% \\
(p=1.00)\end{array}$ \\
\hline Sherman et al. ${ }^{18}$ & NRT & Not informed & Not informed & No. Intervention group: 44\% Control group: 44\% \\
\hline Richter et al. ${ }^{7}$ & $\begin{array}{l}\text { NRT - During hos- } \\
\text { pitalization and pre- } \\
\text { scribed after discharge }\end{array}$ & & Not informed & $\begin{array}{l}\text { No } \\
\text { Intervention group: } 23 \% \text { Control group: } 26 \% \\
(p=0.23)\end{array}$ \\
\hline Rigotti et al. ${ }^{19}$ & $\begin{array}{l}\text { NRT } \\
\text { Bupropion Vareni- } \\
\text { cline, (Isolated or } \\
\text { combined) }\end{array}$ & Not informed & $\begin{array}{l}\text { Up to } 90 \text { days. } \\
\text {-Control group: } \\
\text { Non-specified }\end{array}$ & $\begin{array}{l}\text { No. Intervention group: } 83.7 \% \text { - Control group: } 60.6 \% \\
\text { RR } 1.38 \text { [95\% Cl, 1.29-1.48]; }(p<0.001)\end{array}$ \\
\hline Thomas et al. ${ }^{20}$ & $\begin{array}{l}\text { NRT } \\
\text { Bupropion Varenicline } \\
\text { After discharge, these } \\
\text { same medications } \\
\text { were offered for } 28 \\
\text { days in a co-participa- } \\
\text { tion regime. }\end{array}$ & Not informed & $\begin{array}{l}\text { During hospital- } \\
\text { ization and for at } \\
\text { least } 28 \text { days after } \\
\text { discharge }\end{array}$ & $\begin{array}{l}\text { Yes } \\
\text { Intervention group: } 43.1 \% \\
\text { Control group: } 28.8 \% \\
(p<0.001)\end{array}$ \\
\hline Busch et al. ${ }^{6}$ & NRT - Patches & $\begin{array}{l}14 \text { to } 21 \mathrm{mg} \text { accord- } \\
\text { ing to the number } \\
\text { of cigarettes } \\
\text { smoked }\end{array}$ & 08 weeks & $\begin{array}{l}\text { Intervention group: } 67.9 \% \\
\text { Control Group: } 58.1 \% \\
\text { (p value not informed) }\end{array}$ \\
\hline Cruvinel $^{21}$ & NRT & $\begin{array}{l}14 \text { to } 21 \text { mg accord- } \\
\text { ing to the number } \\
\text { of cigarettes } \\
\text { smoked }\end{array}$ & 04 weeks & $\begin{array}{l}\text { No } \\
\text { Intervention group: } 27.3 \% \\
\text { Control Group: } 36.4 \% \\
(p=0.44)\end{array}$ \\
\hline
\end{tabular}

NRT: Nicotine Replacement Therapy.

the absence of a statistically significant difference in the responses between the intervention and control groups in these studies. Control groups received interventions already established in the literature as effective, therefore, the standard of effectiveness of experimental treatments was not strong enough for differences to be highlighted. Based on the importance achieved by quitline, studies also seek to find ways to improve adherence to these programs. One of the mechanisms presented was the intermediation, by a researcher, in the participant's access and registration to the quitline, in order to, with this, favor the cessation in post-discharge $\mathrm{e}^{7,17}$.

The studies that showed the efficacy of alternative strategies did not support such a difference when biochemical confirmation criteria were used, demonstrating the fragility of the information provided by self-report ${ }^{11,19,21}$. The exception was a study ${ }^{14}$ that used interactive voice messages and guaranteed medication for a period of up to 90 days after hospital discharge. 
TABLE 3. RISKS OF BIAS OF EACH STUDY.

\begin{tabular}{|c|c|c|c|c|c|c|c|}
\hline \multirow{2}{*}{$\begin{array}{l}\text { Study/Areas } \\
\text { of Bias }\end{array}$} & \multicolumn{2}{|l|}{ Selection } & \multirow{2}{*}{$\begin{array}{l}\text { Perfor- } \\
\text { mance }\end{array}$} & \multirow{2}{*}{$\begin{array}{l}\text { De- } \\
\text { tec- } \\
\text { tion }\end{array}$} & \multirow{2}{*}{$\begin{array}{l}\text { Fric- } \\
\text { tion }\end{array}$} & \multirow{2}{*}{$\begin{array}{l}\text { Re- } \\
\text { port }\end{array}$} & \multirow[t]{2}{*}{ Other/main limitations mentioned by authors } \\
\hline & $\begin{array}{l}\text { Random } \\
\text { sequence }\end{array}$ & $\begin{array}{l}\text { Blinded Allo- } \\
\text { cation }\end{array}$ & & & & & \\
\hline $\begin{array}{l}\text { Dornelas et al } \\
2000\end{array}$ & B & I & $A^{*}$ & I & B & B & $\begin{array}{l}\text { The outcome of cessation was assessed by self-re- } \\
\text { port. }\end{array}$ \\
\hline $\begin{array}{l}\text { Henrikus et al } \\
2005\end{array}$ & B & I & $A^{*}$ & I & B & $\mathrm{B}$ & $\begin{array}{l}\text { The absence of medication supply } \\
\text { is reported by the authors as a possible factor of } \\
\text { interference in the prevalence of cessation. }\end{array}$ \\
\hline $\begin{array}{l}\text { Reid et al } \\
2007\end{array}$ & B & I & $A^{*}$ & I & B & B & $\begin{array}{l}\text { Pilot study, data analysis should be carried out with } \\
\text { caution. }\end{array}$ \\
\hline $\begin{array}{l}\text { Regan et al } \\
2011\end{array}$ & B & B & $A^{*}$ & $\mathrm{~B}$ & B & B & $\begin{array}{l}\text { No biochemical evaluation of cessation at } 1 \text { and } 3 \\
\text { months. } \\
\text { The impossibility of separating the contributions of } \\
\text { voice calls and medication in the treatment effect. } \\
\text { Only patients willing to quit smoking after discharge } \\
\text { were evaluated. }\end{array}$ \\
\hline $\begin{array}{l}\text { Rigotti et al } \\
2014\end{array}$ & B & B & $A^{*}$ & I & B & B & $\begin{array}{l}\text { Single center and it was not possible to separate the } \\
\text { effect of medication from the effect of the RIV. }\end{array}$ \\
\hline $\begin{array}{l}\text { Cummins et al } \\
2016\end{array}$ & $B$ & I & $A^{*}$ & $B$ & $B$ & $B$ & $\begin{array}{l}\text { Over } 90 \% \text { of the patients who were hospitalized and } \\
\text { smokers were not included in the study due to limit- } \\
\text { ed resources (harming the generalization of results). } \\
\text { Only smokers interested in quitting were evaluated. } \\
\text { The biochemical confirmation of cessation by co- } \\
\text { tinine occurred in only } 57 \% \text { of the participants. }\end{array}$ \\
\hline $\begin{array}{l}\text { Fellows et al } \\
2016\end{array}$ & $B$ & $B$ & $A^{*}$ & $B$ & B & $B$ & $\begin{array}{l}\text { Low level of recruitment in two of the hospitals } \\
\text { Follow up with various strategies. }\end{array}$ \\
\hline $\begin{array}{l}\text { Harrington et al } \\
2016\end{array}$ & B & $B$ & $A^{*}$ & $B$ & A & $B$ & $\begin{array}{l}\text { Low adherence to the strategy provided to the } \\
\text { intervention group. }\end{array}$ \\
\hline \multirow[t]{2}{*}{$\begin{array}{l}\text { Sherman et al } \\
2016\end{array}$} & $B$ & $B$ & $A^{*}$ & I & B & $B$ & $\begin{array}{l}\text { Self-reporting with a high tendency of the sample } \\
\text { providing unreliable reports when biochemical } \\
\text { validation (cotinine) was performed. }\end{array}$ \\
\hline & & & & & & & $\begin{array}{l}\text { Counseling in the intervention group was carried out } \\
\text { by a trained researcher and not by a member of the } \\
\text { healthcare team. }\end{array}$ \\
\hline $\begin{array}{l}\text { Richter et al } \\
2016\end{array}$ & $B$ & $B$ & $A^{*}$ & $B$ & B & B & $\begin{array}{l}\text { Counseling in the intervention group was initial- } \\
\text { ly provided the hospital environment, which is } \\
\text { turbulent }\end{array}$ \\
\hline $\begin{array}{l}\text { Rigotti et al } \\
2016\end{array}$ & $B$ & $B$ & $A^{*}$ & $A$ & B & $B$ & $\begin{array}{l}\text { Cessation validity } \\
\text { Biochemically only for the six-month outcome } \\
\text { It was not possible to separate the effect of the } \\
\text { medication from the effect of interactive voice calls. } \\
\text { Applicable only to hospitalized patients who wished } \\
\text { to stop smoking and accepted medication use. } \\
\text { Losses of } 25 \% \text { of follow up in both groups; } 31 \% \text { did } \\
\text { not provide saliva for the } \\
\text { biochemical confirmation of cessation. In those } \\
\text { who did provide it, cessation was not confirmed in } \\
27 \% \text { of the sample }\end{array}$ \\
\hline $\begin{array}{l}\text { Thomas et al } \\
2016\end{array}$ & $B$ & $B$ & $A^{*}$ & $B$ & A & $B$ & $\begin{array}{l}\text { Possible contamination of } \\
\text { interventions in the control group performed by } \\
\text { pharmacists trained. } \\
\text { Standard care varied in the different hospitals } \\
\text { studied }\end{array}$ \\
\hline $\begin{array}{l}\text { Busch et al } \\
2017\end{array}$ & $B$ & $B$ & $A^{*}$ & $B$ & B & $B$ & $\begin{array}{l}\text { A } \\
\text { Pilot study, results analysis should be carried out } \\
\text { with caution. }\end{array}$ \\
\hline Cruvinel 2018 & $B$ & $B$ & $A^{*}$ & $B$ & $B$ & $B$ & $\begin{array}{l}\text { Pilot study, results analysis should be carried out } \\
\text { with caution.. }\end{array}$ \\
\hline
\end{tabular}

B: low risk of bias; A: high risk of bias, I: uncertain risk of bias. " Based on the nature of the studies, the bias (performance) is high, but cannot be changed due to the impossibility of blinding who receives or provides the intervention.

The follow-up of hospitalized patients still poses great challenges. Understanding the cause of early relapses has been the subject of analysis, demonstrating that relapse is related to factors such as continue to smoke during hospitalization, low self-efficacy, depression, greater dependence on nicotine and not setting a date to quit smoking ${ }^{27}$. The high number of losses in the post-discharge follow-up is another 
obstacle in conducting treatment, even with the help of modern communication technologies. The difficulty of follow-up and, therefore, of offering the intervention is pointed out in one of the studies as a justification for why established strategies, such as pharmacotherapy and telephone counseling, have failed to demonstrate the expected result in clinical trials ${ }^{15}$.

Some authors consider that the negative result of the intervention does not necessarily invalidate it, and it may be necessary to have a better understanding of in which contexts they would be the most effective options ${ }^{7}$.

This study has limitations related to the barriers of working with control groups that are not exempt from intervention, due, in fact, to the ethical implications imposed on this issue.

\section{CONCLUSION}

The idea that cessation should be promoted at every opportunity to approach smokers reinforces the need to build and apply intervention protocols for hospitalized smokers. The time of hospitalization is an especially opportune occasion for the treatment of smoking.
Pharmacotherapy has been confirmed as an important element in promoting cessation in hospitalized smoking patients. The important role of communication technologies in the monitoring of the patient after discharge was also highlighted.

In Brasil, the population's growing access to cell phones makes the use of communication technologies very promising for the monitoring of smoking patients. It is still a great challenge for future studies to improve technologies to adapt to the social and economic realities of the Brasilian context.

\section{Authors' contribution}

Lígia Menezes do Amaral: Conception and planning of the work, interpretation of the evidence, data collection, writing, revision of the preliminary and final versions; Ângela C. D. Albino Destro de Macêdo: Data collection and writing; Isabella Oliveira Lanzieri: Data collection and writing; Rafaela de Oliveira Andrade: Data collection and writing; Kimber P. Richter: Writing, revision of the preliminary and final versions and approval of the final version; Isabel C. Gonçalves Leite: Design and planning of the work, interpretation of the evidence, data collection, writing, review of the preliminary and final versions and approval of the final version.

\section{RESUMO}

OBJETIVO: O objetivo deste estudo foi avaliar as estratégias no acompanhamento pós-alta para a promoção da cessação no paciente tabagista hospitalizado.

MÉTODOS: Foi realizada uma revisão sistemática tomando-se por referência o protocolo Preferred Reporting Itens for Systematic Rewiews and Meta-Analyses (Prisma-P). Foram utilizadas as seguintes bases de dados: PubMed, Lilacs/Bireme, Scopus, Web of Science, Cochrane e SciELO. Os artigos incluídos foram ensaios clínicos randomizados, publicados entre 1990 e 2018, que promoveram intervenções durante e após a alta hospitalar, intervenções essas que se mantiveram pelo período mínimo de 30 dias após a alta. Os estudos deveriam ter como desfecho a avaliação da cessação do tabagismo.

RESULTADOS: Quatorze estudos foram selecionados para a análise. A revisão dos artigos destacou a farmacoterapia como elemento importante para a promoção da cessação, bem como o uso das novas tecnologias de comunicação no acesso pós-alta.

CONCLUSÃo: Ainda se impõe como um desafio o aprimoramento das estratégias de follow-up após a alta hospitalar para se adequarem aos contextos locais e alcançarem melhores taxas de cessação.

PALAVRAS-ChAVE: Abandono do uso de tabaco. Abandono do hábito de fumar. Alta do paciente. Hospitalização. Revisão sistemática.

\section{REFERENCES}

1. Brasil. Ministério da Saúde. TabNet Win32 3.0: Procedimentos hospitalares do SUS - por local de internação - Brasil [Internet]. 2018 [cited 2019 Nov 11]. Available from: http://tabnet.datasus.gov.br/cgi/tabcgi.exe?sih/cnv/qiuf.def

2. Stead LF, Perera R, Bullen C, Mant D, Hartmann-Boyce J, Cahill K, et al. Nicotine replacement therapy for smoking cessation. Cochrane Database Syst Rev. 2012;11:CD000146.

3. Rigotti NA, Clair C, Munafò MR, Stead LF. Interventions for smoking cessation in hospitalised patients. Cochrane Database Syst Rev. 2012;(5):CD001837.

4. Richter KP, Faseru B, Mussulman LM, Ellerbeck EF, Shireman TI, Hunt
IJ, et al. Using "warm handoffs" to link hospitalized smokers with tobacco treatment after discharge: study protocol of a randomized controlled trial. Trials. 2012;13:127.

5. Moher D, Shamseer L, Clarke M, Ghersi D, Liberati A, Petticrew M, et al. Preferred reporting items for systematic review and meta-analysis protocols (PRISMA-P) 2015 statement. Syst Rev. 2015;4:1.

6. Busch AM, Tooley EM, Dunsiger S, Chattillion EA, Srour JF, Pagoto SL, et al. Behavioral activation for smoking cessation and mood management following a cardiac event: results of a pilot randomized controlled trial. BMC Public Health. 2017;17(1):323. 
7. Richter KP, Faseru B, Shireman TI, Mussulman LM, Nazir N, Bush T, et al. Warm handoff versus fax referral for linking hospitalized smokers to quitlines. Am | Prev Med. 2016;51(4):587-96.

8. Higgins |, Thomas |, Chandler |, Cumpston M, Li T, Page M, et al., eds. Cochrane handbook for systematic reviews of interventions. Chichester: John Wiley \& Sons; 2011

9. Carvalho APV, Silva V, Grande AJ. Avaliação do risco de viés de ensaios clínicos randomizados pela ferramenta da colaboração Cochrane. Diagn Tratamento. 2013;18(1):38-44.

10. Dornelas EA, Sampson RA, Gray JF, Waters D, Thompson PD. A randomized controlled trial of smoking cessation counseling after myocardial infarction. Prev Med. 2000;30(4):261-8.

11. Hennrikus D|, Lando HA, McCarty MC, Klevan D, Holtan N, Huebsch JA, et al. The TEAM project: the effectiveness of smoking cessation intervention with hospital patients. Prev Med. 2005;40(3):249-58.

12. Reid RD, Pipe AL, Quinlan B, Oda J. Interactive voice response telephony to promote smoking cessation in patients with heart disease: a pilot study. Patient Educ Couns. 2007:66(3):319-26.

13. Regan S, Reyen M, Lockhart AC, Richards AE, Rigotti NA. An interactive voice response system to continue a hospital-based smoking cessation intervention after discharge. Nicotine Tob Res. 2011;13(4):255-60.

14. Rigotti NA, Regan S, Levy DE, Japuntich S, Chang Y, Park ER, et al. Sustained care intervention and postdischarge smoking cessation among hospitalized adults: a randomized clinical trial. JAMA. 2014;312(7):719-28.

15. Cummins SE, Gamst AC, Brandstein K, Seymann GB, Klonoff-Cohen H, Kirby CA, et al. Helping hospitalized smokers: a factorial RCT of nicotine patches and counseling. Am J Prev Med. 2016;51(4):578-86.

16. Fellows |L, Mularski RA, Leo MC, Bentz Cl, Waiwaiole LA, Francisco MC, et al. Referring hospitalized smokers to outpatient quit services: a randomized trial. Am J Prev Med. 2016;51(4):609-19.

17. Harrington KF, Kim Y-I, Chen M, Sadasivam RS, Houston TK, Bailey WC, et al. Web-based intervention for transitioning smokers from inpatient to outpatient care: an RCT. Am J Prev Med. 2016;51(4):620-9.

18. Sherman SE, Link AR, Rogers ES, Krebs P, Ladapo JA, Shelley DR, et al. Smoking-cessation interventions for urban hospital patients: a randomized comparative effectiveness trial. Am J Prev Med. 2016;51(4):566-77.

19. Rigotti NA, Tindle HA, Regan S, Levy DE, Chang Y, Carpenter KM, et al. A post-discharge smoking-cessation intervention for hospital patients. Am J Prev Med. 2016;51(4):597-608.

20. Thomas D, Abramson MI, Bonevski B, Taylor S, Poole SG, Paul E, et al. Integrating smoking cessation into routine care in hospitals: a randomized controlled trial. Addiction. 2016;111(4):714-23.

21. Cruvinel E. Mensagens de texto e aconselhamento por telefone como suporte à cessação tabágica entre fumantes em alta hospitalar: um estudo clínico de viabilidade [Tese de doutorado]. Juiz de Fora: Universidade Federal de Juiz de Fora; 2016.

22. Stead LF, Perera R, Lancaster T. A systematic review of interventions for smokers who contact quitlines. Tob Control. 2007;16(Suppl 1):i3-8.

23. Lancaster T, Stead LF. Individual behavioural counselling for smoking cessation. Cochrane Database Syst Rev. 2017;3:CD001292.

24. Abu-Hasaballah K, James A, Aseltine RH Jr. Lessons and pitfalls of interactive voice response in medical research. Contemp Clin Trials. 2007;28(5):593-602.

25. Clinical Practice Guideline Treating Tobacco Use and Dependence 2008 Update Panel, Liaisons, and Staff. A clinical practice guideline for treating tobacco use and dependence: 2008 update. A U.S. Public Health Service report. Am | Prev Med. 2008:35(2):158-76.

26. Zhu SH, Anderson CM, Tedeschi G), Rosbrook B, Johnson CE, Byrd M, et al. Evidence of real-world effectiveness of a telephone quitline for smokers. N Engl | Med. 2002;347(14):1087-93.

27. Mussulman LM, Scheuermann TS, Faseru B, Nazir N, Richter KP. Rapid relapse to smoking following hospital discharge. Prev Med Rep. 2019;15:100891. 